International Journal of Computer Science \& Information Technology (IJCSIT), Vol 2, No 6, December 2010

\title{
SEGMENTATION AND OBJECT RECOGNITION USING EDGE DETECTION TECHNIQUES
}

\author{
Y.Ramadevi, T.Sridevi, B.Poornima, B.Kalyani \\ Department of CSE, Chaitanya Bharathi Institute of Technology \\ Gandipet, Hyderabad. \\ yrdecbit.ac.in
}

\begin{abstract}
Image segmentation is to partition an image into meaningful regions with respect to a particular application. Object recognition is the task of finding a given object in an image or video sequence. In this paper, interaction between image segmentation (using different edge detection methods) and object recognition are discussed. Edge detection methods such as Sobel, Prewitt, Roberts, Canny, Laplacian of Guassian(LoG) are used for segmenting the image. Expectation-Maximization (EM) algorithm, OSTU and Genetic algorithms were used to demonstrate the synergy between the segmented images and object recognition.
\end{abstract}

\section{KEYWORDS}

EM algorithm, OSTU, Genetic Algorithm, Image Segmentation, Object Recognition.

\section{INTRODUCTION}

Image segmentation is the foundation of object recognition and computer vision. In general, image noise should be eliminated through image preprocessing. And there is some specificallygiven work (such as region extraction and image marking) to do after the main operation of image segmentation for the sake of getting better visual effect. Two major computer vision problems, image segmentation and object recognition, have been traditionally dealt with using a strict, bottom-up ordering.

Image segmentation is the process of partitioning/subdividing a digital image into multiple meaningful regions or sets of pixels regions with respect to a particular application. The segmentation is based on measurements taken from the image and might be grey level, colour, texture, depth or motion. The result of image segmentation is a set of segments that collectively cover the entire image. All the pixels in region are similar with respect to some characteristic or computed property, such as color, intensity, or texture. Adjacent regions differ with respect to same characteristics. Edge detection is one of the frequently used techniques in digital image processing.

Object recognition is the task of finding a given object in an image or video sequence. For any object in an image, there are many 'features' which are interesting points on the object that can be extracted to provide a "feature" description of the object. This description extracted from a training image can then be used to identify the object when attempting to locate the object in a test image containing many other objects[3][4].

Image segmentation is done using various edge detection techniques such as Sobel, Prewitt, Roberts, Canny, LoG, EM algorithm, OSTU and Genetic Algorithm [1][2]. Implementation 
between Segmentation and Object recognition using the said techniques is studied [1]. Experimentation is performed on gray scale image using MATLAB 7.9. In this paper, Section 2 discusses about segmentation, and comparison of various edge detection techniques. Section 3 discusses about image segmentation and object recognition using other techniques. The implementation and results are shown in section 4 . The paper is concluded in section 5 .

\section{SEGMENTATION}

Segmentation is the process of partitioning a digital image into its constituent parts or objects or regions. These regions share common characteristics based on color, intensity, texture, etc. The first step in image analysis is to segment an image based on discontinuity detection technique (Edge-based) or similarity detection technique (Region-based). In discontinuity detection technique, one approach is to partition an image based on abrupt changes in intensity near the edges and it is known as Edge-based segmentation. In Similarity detection technique, regionbased segmentation partitions an image into regions that are similar according to a set of predefined criteria. Region-based segmentation looks for uniformity within a sub-region, based on a desired property, e.g., intensity, color, and texture as shown figure 1. The difference between Region-based segmentation and edge-based segmentation is shown in Table 1.

Active contours are popular technique for image segmentation. An advantage of active contours as image segmentation methods is that they partition an image into sub-regions with continuous boundaries. There are two kinds of active contour models: Edge-based active contours use an edge detector, usually based on the image gradient, to find the boundaries of sub-regions and to attract the contours to the detected boundaries. Region-based active contours use the statistical information of image intensity within each subset instead of searching geometrical boundaries

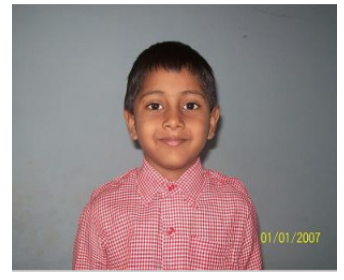

\section{Original Image}

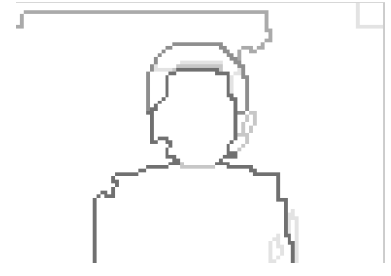

Region Based Segmentation Figure 1. Region based Segmentation

Table 1. Differences between Region-based segmentation and edge-based segmentation

\begin{tabular}{|l|l|}
\hline Region-based segmentation & Edge based segmentation \\
\hline Closed boundaries & Boundaries formed not necessarily closed \\
\hline $\begin{array}{l}\text { Multi-spectral images improve } \\
\text { segmentation }\end{array}$ & $\begin{array}{l}\text { No significant improvement for multi-spectral } \\
\text { images }\end{array}$ \\
\hline Computation based on similarity & Computation based on difference \\
\hline
\end{tabular}

\subsection{Edge Detection for Image Segmentation}

An Edge in an image is a significant local change in the image intensity, usually associated with a discontinuity in either the image intensity or the first derivative of the image intensity. The three steps in Edge detection process is[6] a) Filtering b) Enhancement and c) Detection. 
a. Filtering: Images are corrupted by noise such as salt and pepper noise, impulse noise and Gaussian noise. As there is a trade-off between edge strength and noise reduction, filtering is done.

b. Enhancement: It emphasizes pixels where there is a significant change in local intensity values and is usually performed by computing the gradient magnitude.

c. Detection: Many points in an image have a nonzero value for the gradient, and not all of these points are edges for a particular application. Thresholding is used for the detection of edge points.

The different edge detection methods used are Sobel, Prewitt, Roberts, Canny, LoG, EM algorithm, OSTU algorithm and Genetic Algorithm.

2.1.1. Sobel Operator: It performs $2-D$ spatial gradient measurement on an image and so emphasizes regions of high spatial frequency that correspond to edges. The convolution mask of Sobel operator are as shown Figure 2, which are used to obtain the gradient magnitude of the image from the original

\begin{tabular}{|c|l|l|}
\hline 1 & 2 & 1 \\
\hline 0 & 0 & 0 \\
\hline-1 & -2 & -1 \\
\hline
\end{tabular}

\begin{tabular}{|l|l|l|}
\hline-1 & 0 & 1 \\
\hline-2 & 0 & 2 \\
\hline-1 & 0 & 1 \\
\hline
\end{tabular}

Figure 2. Sobel Mask

2.1.2. Prewitt Operator: The prewitt operator is an approximate way to estimate the magnitude and orientation of the edge. The convolution mask of prewitt operator is shown in figure 3.

\begin{tabular}{|l|l|l|}
\hline 1 & 1 & 1 \\
\hline 0 & 0 & 0 \\
\hline-1 & -1 & -1 \\
\hline
\end{tabular}

\begin{tabular}{|l|l|l|}
\hline-1 & 0 & 1 \\
\hline-1 & 0 & 1 \\
\hline-1 & 0 & 1 \\
\hline
\end{tabular}

\section{Figure 3. Prewitt Mask}

2.1.3. Roberts Operator: It performs 2 -D spatial gradient measurement on an image. It highlights regions of high spatial frequency which often correspond to edges. The cross convolution mask is shown in figure 4.

\begin{tabular}{|l|l|}
\hline-1 & 0 \\
\hline 0 & -1 \\
\hline
\end{tabular}

$$
\begin{array}{|l|l|}
\hline 0 & 1 \\
\hline-1 & 0 \\
\hline
\end{array}
$$

Figure 4. Roberts Mask

2.1.4. Laplacian of Guassian(LoG) Operator: It is a second order derivative. The digital implementation of the Laplacian function is made using the mask given in figure 5.

\begin{tabular}{|c|c|c|}
\hline 0 & -1 & 0 \\
\hline-1 & 4 & -1 \\
\hline 0 & -1 & 0 \\
\hline
\end{tabular}

Figure 5. Laplacian of Guassian(LoG) Operator 
2.1.5. Canny Operator: It is a method to find edges by isolating noise from the image without affecting the features of the edges in the image and then applying the tendency to find the edges and the critical value for threshold.

\section{Image Segmentation \& ObJect Recognition}

In Image Segmentation the generative models are used to decide which part of the image a model should occupy. Active Appearance Model (AAMs) is used as generative models and addresses the problem of jointly detecting and segmenting objects in images [1][5]. Regarding recognition, each object hypothesis is validated based on the image area assigned to the object, as well as the estimated model parameters, which indicate the familiarity of the object appearance. On one hand, knowing the area occupied by an object is needed for the estimation of the model parameters and, on the other hand, the model synthesis is used to assign observations to the model. Since neither is known in advance, we cannot address each problem separately. We view this problem as an instance of the broader problem of parameter estimation with missing data: In our case, the missing data are the assignments of observations to models. A well-known tool for addressing such problems is the EM algorithm.

An expectation-maximization (EM) algorithm is used for finding maximum likelihood estimates of parameters in probabilistic models, where the model depends on unobserved latent variables. In order to find maximum likelihood estimate we have to find probability density function and loglikelihood.

\subsection{The Expectation-Maximization Algorithm}

The EM algorithm is an efficient iterative procedure to compute the Maximum Likelihood Estimate (MLE) in the presence of missing or hidden data. Each iteration of the EM algorithm consists of two processes: The E-step, and the M-step. In the expectation, or E-step, the missing data are estimated given the observed data and current estimate of the model parameters. This is achieved using the conditional expectation, explaining the choice of terminology. In the M-step, the likelihood function is maximized under the assumption that the missing data are known. The estimates of the missing data from the E-step are used in lieu of the actual missing data.

The EM algorithm seeks to find the MLE by iteratively applying the following two steps:

Expectation step: Calculate the expected value of the log likelihood function, with respect to the conditional distribution of $z$ given $x$ under the current estimate of the parameters $\theta^{(t)}$.

Maximization step: Find the parameter which maximizes this quantity.

\subsection{Image segmentation using OTSU}

Threshold selection is used in OTSU algorithm. The OTSU method is one of the applied methods of image segmentation in selecting threshold automatically for its simple calculation and good adaptation.

In image processing, OTSU's thresholding method is used for automatic binarization level decision, based on the shape of the histogram. The algorithm assumes that the image is composed of two basic classes: Foreground and Background. It then computes an optimal threshold value that minimizes the weighted within class variances of these two classes. It is 
mathematically proven that minimizing the within class variance is same as maximizing the between class variance.

The different thresholding techniques are

1. Histogram shape-based methods.

2. Clustering-based methods.

3. Entropy-based methods.

4. Object attribute-based method.

5. Spatial methods.

6. Local methods.

The major problem with thresholding is that we consider only the intensity, not any relationships between the pixels. There is no guarantee that the pixels identified by the thresholding process are contiguous.

The steps of the OTSU algorithm: For each potential threshold $\mathbf{T}$,

1. Separate the pixels into two clusters according to the threshold.

2. Find the mean of each cluster.

3. Square the difference between the means.

4. Multiply by the number of pixels in one cluster times the number in the other.

\subsection{Image Segmentation using Genetic Algorithm}

Genetic Algorithms (GAs) can be seen as a software tool that tries to find structure in data that might seem random, or to make a seemingly unsolvable problem more or less 'solvable'. GAs can be applied to domains about which there is insufficient knowledge or the size and/or complexity is too high for analytic solution.

Basically, a genetic algorithm consists of three major operations: selection, crossover, and mutation. The selection evaluates each individual and keeps only the fittest ones in the population. In addition to those fittest individuals, some less fit ones could be selected according to a small probability. The others are removed from the current population. The crossover recombines two individuals to have new ones which might be better. The mutation operator induces changes in a small number of chromosomes units. Its purpose is to maintain the population diversified enough during the optimization process

The existing GA's are founded upon the following main principles:

1. Reproduction

2. Fitness

3. Crossover

4. Mutation

The algorithms code crossover as either a swap of one bit, which is more like a single-point mutation than a 'real crossover', or of several bits (used with genetic programming) and a distinction is made between two parents (bit strings, but called chromosomes in GA terminology) who are the identical, two different parents and single parent. Anyhow, the process has the following procedure: 
1. Select two bit strings (chromosomes), or in case of the genetic programming: select a branch of each parent.

2. Cut the chromosome (or branch) at a particular location.

3. Swap the bits/branches of the two parents.

\section{IMPLEMENTATION AND RESULTS}

Implementation of the techniques was done on different images. Colored images were converted into gray scale image and then segmentation and recognition methods were applied. A sample grey scale image (figure 6) is considered for segmentation and object recognition using Sobel, Prewitt, Roberts, Canny, LoG , EM algorithm, OSTU algorithm and Genetic Algorithm. The segmented image and recognized image using different edge operators' techniques are shown in Table 2 .

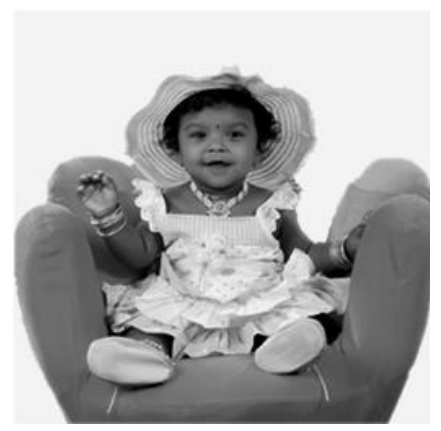

Figure 6. Original Image

Table 2. Segmented and Recognized images using different operators/algorithms

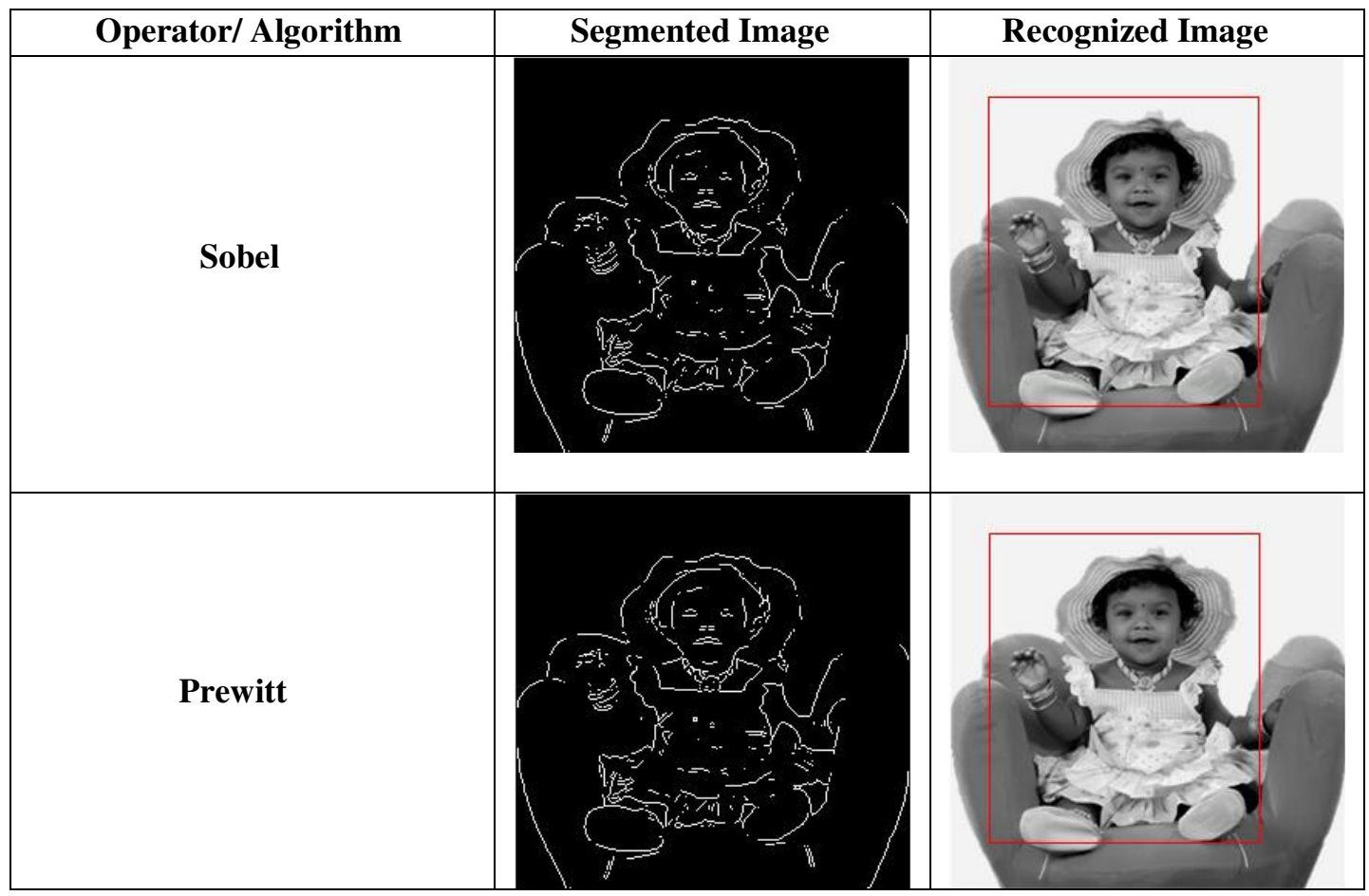


International Journal of Computer Science \& Information Technology (IJCSIT), Vol 2, No 6, December 2010

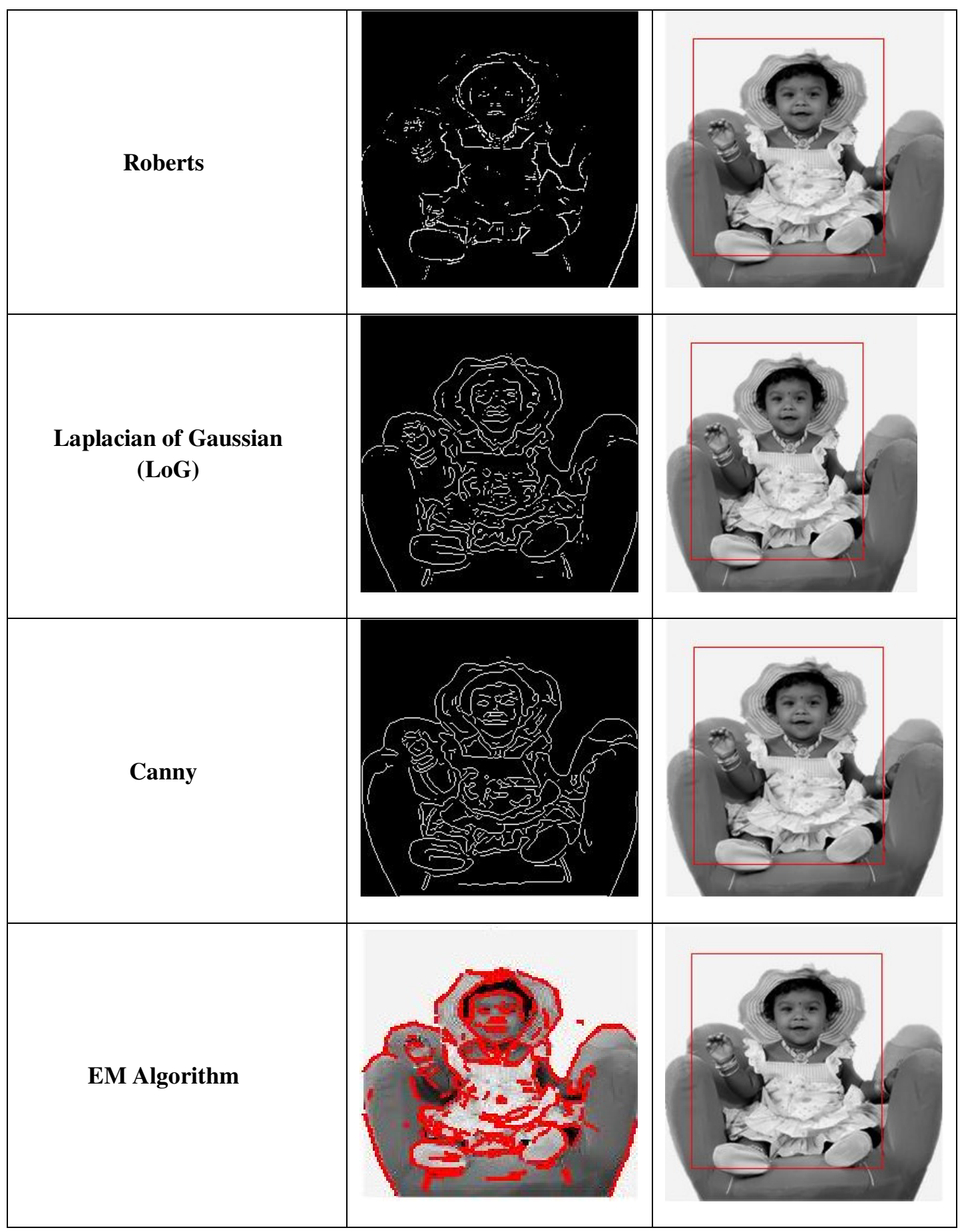




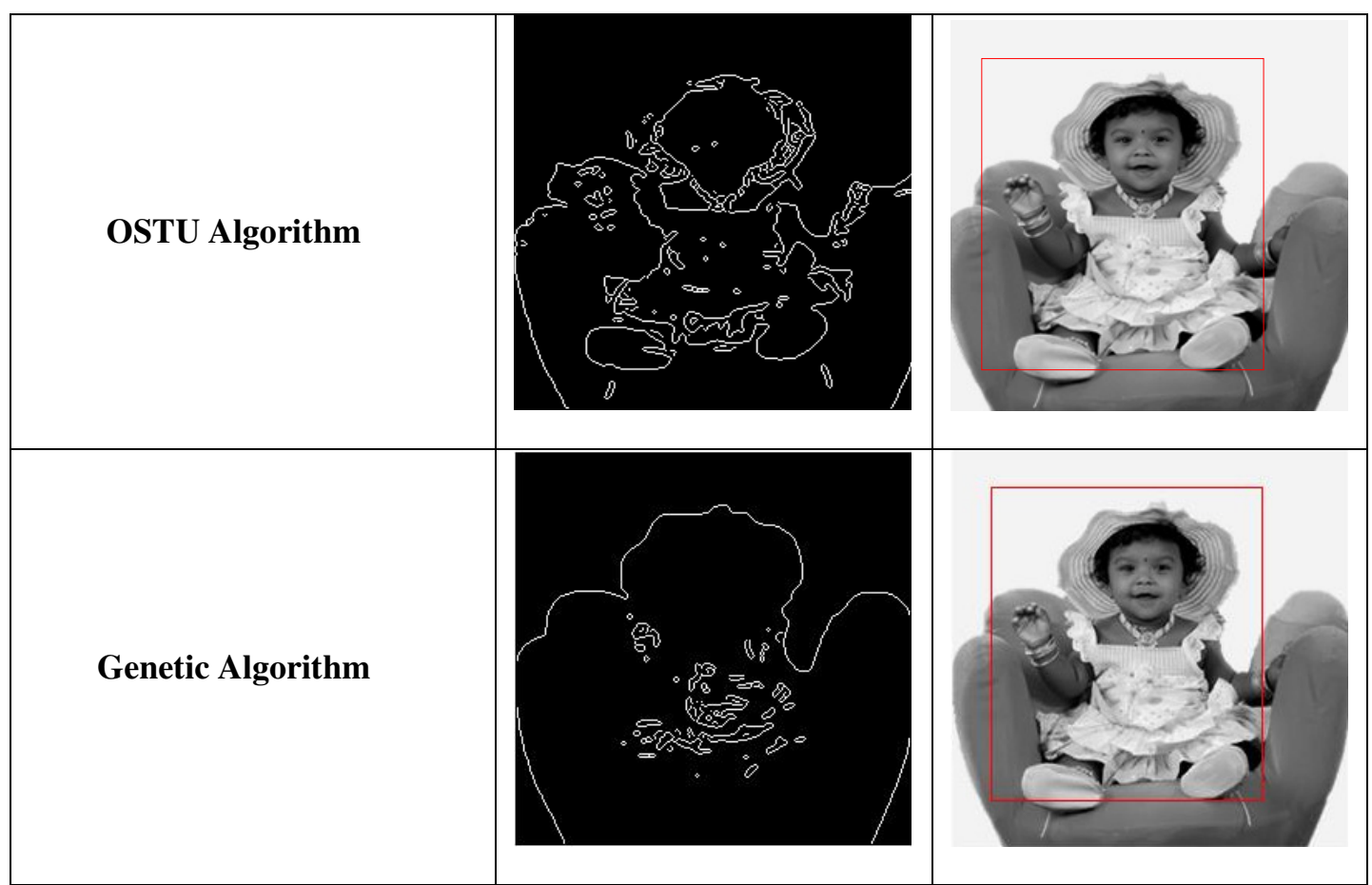

Canny operator performed better than Sobel, Prewitt, Roberts and LoG. The EM algorithm produced stable segmentation effect on different types of images. OTSU showed good and stable segmentation effect. Genetic algorithm exhibited normal segmentation effect on all types of images.

\section{CONCLUSIONS}

This paper focuses mainly on the Image segmentation using edge operators. The interaction between image segmentation and object recognition in the framework of the Sobel, Prewitt, Roberts, Canny, LoG, Expectation-Maximization (EM) algorithm, OSTU Algorithm and Genetic Algorithm are studied. MATLAB 7.9. was used for experimentation image. Expectation-Maximization algorithm and OTSU algorithm exhibited stable segmentation effect.

\section{REFERENCES}

[1] Iasonas Kokkinos, and Petros Maragos (2009),"Synergy between Object Recognition and image segmentation using Expectation and Maximization Algorithm"., IEEE Trans. on Pattern Analysis and Machine Intelligence (PAMI), Vol. 31(8), pp. 1486-1501, 2009.

[2] Wen-Xiong Kang, Qing-Qiang Yang, Run-Peng Liang (2009), "The Comparative Research on Image Segmentation Algorithms," First International Workshop on Education Technology and Computer Science.

[3] V. Ferrari, T. Tuytelaars, and L.V. Gool(2004), "Simultaneous Object Recognition and Segmentation by Image Exploration," Proc. Eighth European Conf. Computer Vision, 2004.

[4] B. Leibe, A. Leonardis, and B. Schiele(2004), "Combined Object Categorization and Segmentation with an Implicit Shape Model," Proc. ECCV Workshop Statistical Learning in Computer Vision, 2004.

[5] Y.Ramadevi, B.Kalyani, T.Sridevi(2010), "Synergy between Object Recognition and Image Segmentation", International Journal on Computer Science and Engineering, Vol. 02, No. 08, 2010, 2767-2772.

[6] N.Senthilkumarn, R.Rajesh(2009), "Edge Detection Techniques for Image Segmentation- A Survey of Soft Computing Approaches", IJRTE, vol1,No2, 2009 250-254. 
International Journal of Computer Science \& Information Technology (IJCSIT), Vol 2, No 6, December 2010

Y Rama Devi received B.E. from Osmania University in 1991 and M.Tech (CSE) degree from JNT University in 1997. She received his Ph.D. degree Central University, Hyderabad in 2009. She is Professor, Chaitanya Bharathi Institute of Technology, Hyderabad. Her research interests include Image Processing, Soft Computing, Data Mining, and Bio-Informatics. She is a member for IEEE, ISTE, IETE, and IE. She has published more than 25 research publications in various National, Inter-national conferences, proceedings and Journals.

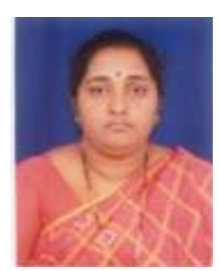

T.Sridevi received B E from Osmania University in 1992 and M.Tech (CSE) degree from JNT University in 2002. She is pursuing her Ph D from Osmania University in Computer Science under the guidance of Dr V. Vijaya Kumar. She has 14 years of teaching/industry experience. She joined as Assistant Professor in Chaitanya Bharathi Institute of Technology, Hyderabad, India in 2002. Presently she is an Associate Professor, Chaitanya Bharathi Institute of Technology, Hyderabad. Her research areas include Water Marking, Image Processing and Soft Computing. She is a life member of IETE. She has published more than 5 research publications in various National, Inter-national conferences, proceedings and Journals.

B.Poornima received her B.Tech from J.N.T.U in 2005. She is pursuing her M.Tech from CBIT under Osmania University. Her area of interest is image processing.

B.Kalyani received B.Sc computers from Sri Krishnadevaraya University, Anantapur in 2003, M.Sc Mathematics from Sri Krishnadevaraya University, Anantapur in 2006 and M.Tech(C.S.E) from Osmania University. Her area of interest is image processing.
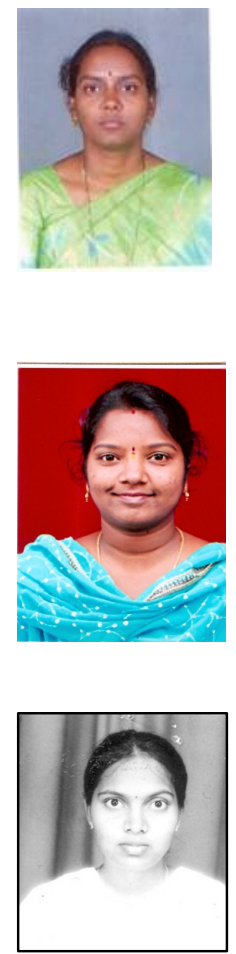